\title{
Routing and Wavelength Assignment in All-Optical Networks
}

\author{
Rajiv Ramaswami, Member, IEEE, and Kumar N. Sivarajan, Member, IEEE
}

\begin{abstract}
This paper considers the problem of routing connections in a reconfigurable optical network using wavelength division multiplexing. Each connection between a pair of nodes in the network is assigned a path through the network and a wavelength on that path, such that connections whose paths share a common link in the network are assigned different wavelengths. We derive an upper bound on the carried traffic of connections (or equivalently, a lower bound on the blocking probability) for any routing and wavelength assignment (RWA) algorithm in such a network. The bound scales with the number of wavelengths and is achieved asymptotically (when a large number of wavelengths is available) by a fixed RWA algorithm. Although computationally intensive, our bound can be used as a metric against which the performance of different RWA algorithms can be compared for networks of moderate size. We illustrate this by comparing the performance of a simple shortest-path RWA (SP-RWA) algorithm via simulation relative to our bound. We also derive a similar bound for optical networks using dynamic wavelength converters, which are equivalent to circuit-switched telephone networks, and compare the two cases for different examples. Finally, we quantify the amount of wavelength reuse achievable in large networks using the SP-RWA via simulation as a function of the number of wavelengths, number of edges, and number of nodes for randomly constructed networks as well as deBruijn networks. We also quantify the difference in wavelength reuse between two different optical node architectures. The results show that it is feasible to provide several all-optical connections to each node in a large network using a limited number of wavelengths. For instance, using 32 wavelengths, it is possible to provide 10 full-duplex connections to each node in a 128-node random network with average degree 4 , and 5 full-duplex connections per node in a 10W-node random network with average degree 4 . The results also show that wavelength converters offer a $10-40 \%$ increase in the amount of reuse achievable for our sampling of 14 networks ranging from 16 to 1000 nodes when the number of wavelengths available is small ( 10 or 32 ).
\end{abstract}

\section{INTRODUCTION}

$\mathbf{W}$ AVELENGTH-division-multiplexing (WDM) technology offers the capability of building very large widearea networks consisting of thousands of nodes with per-node throughputs of the order of a gigabit-per-second [1]-[4]. Fig. 1 shows a WDM all-optical network employing wavelength routing, consisting of optical routing nodes interconnected by optical links. Each link is assumed to be bidirectional

Manuscript received May 3. 1995; revised October 11, 1995; approved by iEEE/ACM Transactions on Networking Editor B. Mukherjee. This work was supported in part by ARPA under Grant MDA 972-92-C-0075.

R. Ramaswami is with IBM T. J. Watson Research Center, Yorktown Heights, NY 10598 USA (e-mail: rajiv@watson.ibm.com).

K. N. Sivarajan is with the Indian Institute of Science, Bangalore, India (email: Kumar@ece.iisc.ernet.in). He is also with the IBM T. J. Watson Research Center, Yorktown Heights, NY 10598 USA.

IEEE L.og Number 9414429

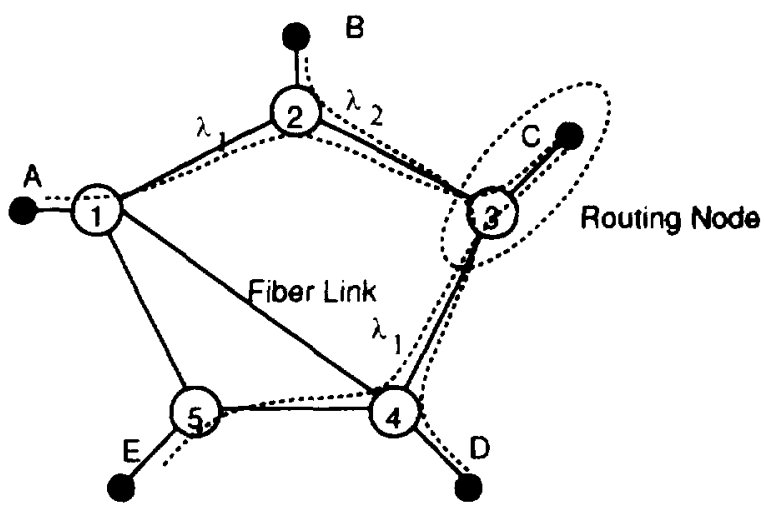

Fig. 1. A WDM network consisting of routing nodes interconnecled by point-to-point fiber-optic links.

and actually consists of a pair of unidirectional links. An optical routing node is capable of routing each wavelength on an incoming link to any outgoing link. However, the same wavelength on two incoming links cannot be routed simultaneously onto a single outgoing link. If there are $\Lambda$ wavelengths on each link, the routing node may be viewed as consisting of $\Lambda$ independent switches, one for each wavelength (Fig. 2). Each switch has $\Delta$ inputs and $\Delta$ outputs where $\Delta$ is the number of incoming (and outgoing) links. There is no optical to electronic conversion and vice versa, and hence no buffering, at the intermediate nodes, in these all-optical networks. In addition to routing and switching signals, the optical node also serves as a source and sink of traffic in the network. Theoretical studies of such networks appear in [5]-[14] and these networks are currently being explored at the testbed level by several groups [3].

In our network model, connection requests and terminations arrive at random. Each connection must be assigned a specific path in the network and a wavelength which is the same on every link on the assigned path. Moreover the wavelengths and paths assigned must be such that no two paths that share an edge are assigned the same wavelength. For example, in Fig. 1, a connection between node $A$ and node $C$ is carried on wavelength $\lambda_{1}$, a connection between node $C$ and node $E$ also on the same wavelength $\lambda_{1}$, but a connection between node $B$ and node $D$ must be carried on a different wavelength $\lambda_{2}$.

This routing and wavelength assignment (RWA) problem, or variants of it, have been considered earlier in [7]-[9]. Several heuristic RWA algorithms have been proposed and their performance has been quantified via simulation.

Related work on determining the number of wavelengths required to support specific traffic patterns, such as permu- 


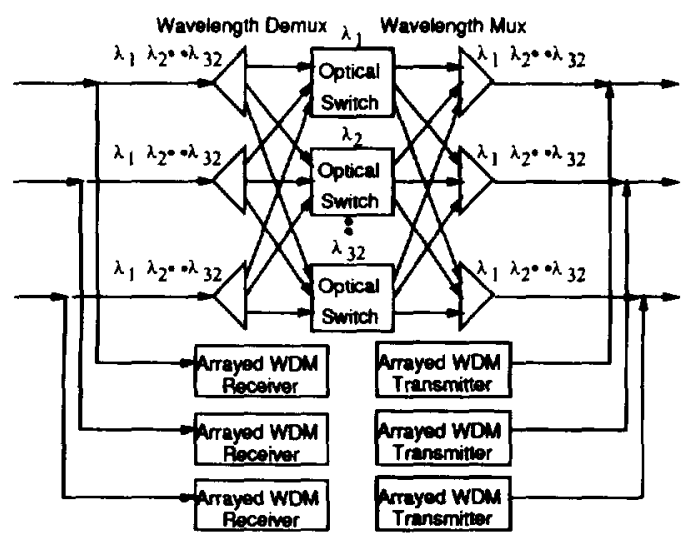

Local Source and Sirk

Fig. 2. Structure of an optical routing node and its attached source and sink of traffic. The routing node can switch each wavelength at its input ports independent of the other wavelengths.

tation routing, and constructing networks using a minimal number of wavelengths appears in [10]-[12]. Related models are also considered in [13], [14] where a "virtual topology" is imposed on top of the underlying physical topology. The virtual topology is a graph consisting of the nodes in the network with an edge between two nodes if a connection is set up between the two nodes using some wavelength and path in the physical topology.

A similar routing problem arises in circuit-switched telephone networks. Here, we must route connections by selecting a path for each connection such that there is a circuit available to accommodate the call in every link on the path. In our optical network model, we must not only satisfy the constraint in circuit-switched telephone networks, but also satisfy an additional constraint that the same circuit (wavelength) must be assigned to the connection on every link in the path. Note that if we are allowed to use dynamic wavelength converters inside the optical network, then it becomes equivalent to the circuit-switched telephone network. We assume that the wavelength converters are used such that a signal at a particular wavelength on an input link can be converted to any other wavelength on any of the output links (as long as two connections do not use the same wavelength on a single link). This can be achieved in principle by using wavelength converters in conjunction with a large switch inside an optical routing node as shown in Fig. 3. This configuration adds significant complexity to the routing-node structure but will yield somewhat better wavelength reuse, as will be seen later. The routing node now has a single wavelength-independent switch with $\Lambda \Delta$ inputs and outputs as compared to $\Lambda$ switches with $\Delta$ inputs and outputs for the case without converters. It is possible to have intermediate structures allowing partial wavelength conversion [15], but we do not consider this in our paper. Henceforth, we will use the term "circuit-switched" to refer to a circuit-switched telephone network or to an optical network using dynamic wavelength converters. We will use the term "optical network" to refer to a network that does not use wavelength converters.

The routing problem in circuit-switched networks has been studied extensively [16], [17]. It is well known that the routing

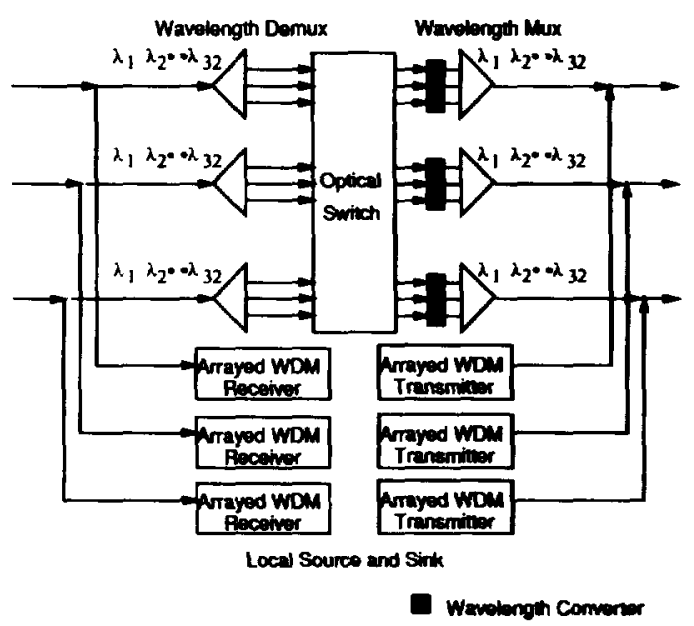

Fig. 3. Structure of an optical routing node with wavelength converters. The node can switch a signal at a particular wavelength on an input port to any wavelength on any output port.

problem can be formulated as an integer linear program (ILP) [18, ch. 6, Section 2]. In [16], it was shown that an upper bound on the carried traffic can be obtained by relaxing the ILP to a linear program (LP), and moreover, that the bound holds for random offered traffic as well. Since the RWA problem in our model is a more constrained version of the routing problem in circuit-switched networks, it is clear that this bound will also be an upper bound for the carried traffic in our network model. However, our objective in this paper is to derive a better upper bound.

For clarity of exposition, we first consider the case when we are given a fixed set of connections to be routed. We formulate the RWA problem as an integer linear program (ILP) where the objective is to maximize the number of connections that are successfully routed. If we relax the integrality constraints in this ILP, we get an LP whose value represents an upper bound on the number of connections that can be successfully routed. We show that a straightforward formulation, when relaxed into a linear program, yields an upper bound that is the same as the upper bound for the circuit-switched case. We then show how to formulate the ILP suitably so as to get a better upper bound when it is relaxed into an LP.

We then consider the more general case where connections arise at random between some source-destination pair in the network and have a random holding time. (The deterministic case is a special case of this more general model.) For this case, using the results in [16], we show the following. By suitably normalizing the LP for the deterministic case by the number of available wavelengths, we obtain an upper bound on the carried traffic (expected number of connections in progress) per available wavelength that is achievable by any RWA algorithm for this network. Moreover, for a large class of traffic models (including the standard Poisson arrivals), there exist RWA algorithms whose carried traffic approaches this upper bound arbitrarily closely when the number of available wavelengths is sufficiently large.

The usefulness of our bound lies in the fact that it can be used as a benchmark against which the performance of various heuristic RWA algorithms can be compared. We illustrate 
this by comparing the simulated performance of a simple shortest-path heuristic RWA (SP-RWA) algorithm against the bound. Moreover the solution to the LP that gives the bound can also indicate how to modify the network to improve its performance.

Finally, we quantify the amount of wavelength reuse achievable in large networks using the SP-RWA algorithm as a function of the number of nodes, edges and wavelengths via simulation. We consider both randomly constructed networks as well as regular deBruijn networks.

The paper is organized as follows. The next section formulates the ILP's and corresponding LP's for the RWA problem. Section III presents two examples that show that this bound is indeed better than the bound for circuit-switched networks, and also compares the performance of the heuristic and a fixed routing algorithm against our bound in one of the examples. Section IV presents simulation results for the wavelength reuse factor as a function of various network parameters. Section $\mathrm{V}$ proposes an alternate routing node architecture that trades off complexity against performance. Section VI discusses the implications of our work and concludes the paper.

\section{THE BOUND}

We represent the network by an undirected graph, $G$. Each node in the graph corresponds to a node in the network and each edge to a link. We assume that all connections to be routed are full duplex, that all links are bidirectional and the two halves of a duplex connection are to be routed over the same path using the same wavelength.

Let $N$ denote the number of source-destination $(s-d)$ pairs in the network, $M$ the number of links and $\Lambda$ the number of wavelengths available on each link (assumed to be the same for all links). For any RWA algorithm, let $m_{i}, i=1, \cdots, N$, denote the number of connections carried between sourcedestination pair $i$, and $m$, the $N$-vector $\left(m_{i}\right)$. Let $\rho$ denote the total offered load. $p_{i}, \quad i=1, \cdots, N$, the offered load between source-destination pair $i$, and $\mu$, the $N$-vector $\left(p_{i}\right)$. The offered load for the deterministic case (Sections Il-A and $-B$ ) is the number of connections that are available to be routed. In the random case (Section II-C), it is the expected number of connections that would be in progress if one could successfully route all call arrivals.

Let $P$ denote the total number of available paths on which connections can be routed. The set of paths could either be given or can be computed given the graph $G$ and the set of source-destination pairs. (Note that the number of paths between a source-destination pair in an arbitrary graph/network can be exponential in the number of nodes or links).

Let $A=\left(a_{i j}\right)$ be the $P \times N$ path-s- $d$-pair incidence matrix, i.e.,

$$
a_{i j}= \begin{cases}1, & \text { if path } i \text { is between source-destination pair } j, \\ 0, & \text { otherwise. }\end{cases}
$$

Let $B=\left(b_{i j}\right)$ be the $P \times M$ path-edge incidence matrix, i.e.,

$$
b_{i j}= \begin{cases}1, & \text { if link } j \text { is on path } i \\ 0, & \text { otherwise. }\end{cases}
$$

We first give a straightforward ILP formulation of the RWA problem as a multicommodity flow problem [18, ch. 6. Section 2].

\section{A. A Simple Formulation}

The operation of every RWA algorithm in an optical network can be represented by a $P \times \Lambda$ path-wavelength assignment matrix which we denote by $C=\left(c_{i j}\right)$ where,

$c_{i j}=$

$\{1$, if the RWA algorithm assigns wavelength $j$ to path $i$.

$\{0$, otherwise.

Then, the optimal RWA algorithm for the deterministic case is found by solving the following ILP whose value we denote by $C_{o}(\rho, p)$.

\section{(Maximize carried traffic)}

$$
C_{o}(\rho, p)=\max \sum_{i=1}^{N} m_{i}
$$

subject to

$$
\begin{aligned}
& m_{i} \geq 0 . \quad \text { integer, } \quad i=1, \cdots, N, \\
& c_{i j} \geq 0, \quad \text { integer. } \quad i=1, \cdots, P, j=1, \cdots, \Lambda \text {, }
\end{aligned}
$$

(capacity constraint: the same wavelength is used at most once on a given link)

$$
C^{T} B \leq 1.1 \times M
$$

(traffic demands)

$$
\begin{aligned}
m & \leq 1_{\Lambda} C^{T} A . \\
m_{i} & \leq p_{i} \rho, \quad i=1, \cdots, N .
\end{aligned}
$$

$\left(1_{X} \times Y\right.$ represents an $X \times Y$ matrix all of whose elements are unity, and $1 x$ represents a $1 \times X$ matrix all of whose elements are unity.)

Now consider the corresponding circuit-switched network. The operation of every routing algorithm in such a network can be represented by a vector of path-flows $f=\left(f_{i}\right)$ where $f_{i}$ denotes the flow on path $i$. Then, the optimal routing algorithm for the deterministic case is found by solving the following ILP whose value we denote by $C_{c}(\rho, p)$.

(Maximize carried traffic)

$$
C_{c}(\rho, p)=\max \sum_{i=1}^{N} m_{i}
$$

subject to

$$
\begin{aligned}
& m_{i} \geq 0, \quad \text { integer, } \quad i=1, \cdots, N \text {, } \\
& f_{i} \geq 0 . \quad \text { integer, } \quad i=1, \cdots, P \text {. }
\end{aligned}
$$

(capacity constraint: not more than $\Lambda$ units of flow on any link)

$$
f B \leq 1_{M} \Lambda
$$

(traffic demands)

$$
\begin{aligned}
m & \leq f A, \\
m_{i} & \leq p_{i} \rho, \quad i=1, \cdots, N .
\end{aligned}
$$


Lemma 1:

$$
C_{o}(\rho, p) \leq C_{c}(\rho, p)
$$

Proof: Let $c_{i j}^{s}$ and $m_{i}^{s}$ constitute a feasible solution to the $C_{o}(\rho, p)$ ILP. Then set $f_{i}=\sum_{j=1}^{\Lambda} c_{i j}^{s}$ and $m_{i}=m_{i}^{s}$. Since $f=1_{\Lambda} C^{T}$,

$$
f A=1_{\Lambda} C^{T} A \geq m
$$

and

$$
f B=1_{\Lambda} C^{T} B \leq 1_{\Lambda} 1_{\Lambda \times M}=1_{M} \Lambda
$$

Therefore, $f_{i}$ and $m_{i}$ constitute a feasible solution to the $C_{c}(\rho, p)$ ILP. Hence,

$$
C_{o}(\rho, p) \leq C_{c}(\rho, p)
$$

In order to get upper bounds on $C_{o}(\rho, p)$ and $C_{c}(\rho, p)$, we can drop the integrality constraints on $m_{i}, c_{i j}$ and $f_{i}$ and solve the corresponding LP's. Let $U_{o}(\rho, p)$ and $U_{c}(\rho, p)$ denote the (maximum) values of the LP's for the optical and circuitswitched case respectively. Then, we can show (with a proof virtually identical to that of Lemma 1), that

$$
U_{o}(\rho, p) \leq U_{c}(\rho, p)
$$

Interestingly however, suppose $f_{i}^{\prime}$ and $m_{i}^{\prime}$ constitute a feasible solution to the $U_{c}(\rho, p)$ LP. Then set $c_{i j}=f_{i}^{\prime} / \Lambda$ and $m_{i}=m_{i}^{\prime}$. Since $C=f^{T} 1_{\Lambda} / \Lambda$,

$$
1_{\Lambda} C^{T} A=1_{\Lambda} 1_{\Lambda}^{T} f A / \Lambda=f A \geq m
$$

and

$$
C^{T} B=1_{\Lambda}^{T} f B / \Lambda \leq 1_{\Lambda}^{T} \Lambda 1_{M} / \Lambda=1_{\Lambda \times M} .
$$

Therefore, $c_{i j}$ and $m_{i}$ constitute a feasible solution to the $U_{o}(\rho, p)$ LP. Thus we have the following lemma.

Lemma 2:

$$
U_{o}(\rho, p) \geq U_{c}(\rho, p)
$$

Using (1) and Lemma 2, we have the following proposition. Proposition 1:

$$
U_{o}(\rho, p)=U_{c}(\rho, p)
$$

Thus this formulation of the RWA problem yields an upper bound on carried traffic that is identical to the upper bound for the corresponding circuit-switched network. However, a different formulation of the problem given below can be used to obtain a better upper bound.

\section{B. A Better Formulation}

We create a new graph $G_{p}$ where each node corresponds to a path in $G$ and two nodes in $G_{p}$ are adjacent iff the corresponding two paths in $G$ share a common link. Now our RWA problem is transformed into assigning wavelengths to nodes in $G_{p}$ so that no two adjacent nodes are assigned the same wavelength. In other words, a set of paths in $G$ can be assigned a common wavelength only if the corresponding nodes in $G_{p}$ form an independent set. Let $L$ denote the number of maximal independent sets in $G_{p}$, let $w_{i}$ denote the number of wavelengths that are assigned to the nodes in independent set $i$ by a RWA algorithm, and $w$ the $L$-vector $\left(w_{i}\right){ }^{\prime}$ Let $D=\left(d_{i j}\right)$ be the $P \times L$ path-independent-set incidence matrix, i.e.,

$$
d_{i j}= \begin{cases}1, & \text { if independent set } j \text { contains path } i, \\ 0, & \text { otherwise. }\end{cases}
$$

The ILP, whose value we denote as $T_{o}^{\Lambda}(\rho, p)$ can then be formulated as follows:

(Maximize carried traffic)

$$
T_{o}^{\Lambda}(\rho, p)=\max \sum_{i=1}^{N} m_{i}
$$

subject to

$$
w_{i} \geq 0, \quad \text { integer, } \quad i=1, \cdots, L,
$$

(capacity constraint-not more than $\Lambda$ units of flow on any link)

$$
\sum_{i=1}^{L} w_{i} \leq \Lambda
$$

(traffic demands)

$$
\begin{aligned}
f & \leq w D^{T}, \\
m & \leq f A, \\
m_{i} & \leq p_{i} \rho, \quad i=1, \cdots, N .
\end{aligned}
$$

\section{Random Traffic Demands}

Henceforth, we let $\rho_{i}$ denote the offered traffic to $s$ - $d$ pair $i$ in Erlangs. ${ }^{2}$ If calls arrive at random and have random holding times, under the operation of any RWA algorithm, the network is in a random state which we denote by $m=$ $\left(m_{1}, m_{2}, \cdots, m_{N}\right)$ where $m_{i}$ is the number of calls in progress between $s-d$ pair $i$. The set of feasible states for this network when the number of available wavelengths is $\Lambda$, which we denote by $S_{\Lambda}$, is the set of all nonnegative integer $N$-vectors $m$ for which the $T_{o}^{\Lambda}(\rho, p)$ ILP is feasible, i.e., $m \in Z^{N}$ if

\footnotetext{
${ }^{1}$ Note that the number of maximal independent sets could be exponential in the number of nodes in $G_{p}$. The maximal independent sets in $G_{p}$ correspond to maximal cardinality cliques in a graph $G_{p}^{\prime}$ consisting of the same set of nodes as $G_{p}$ and all the edges that are not present in $G_{p}$. An algorithm to generate all cliques in a graph is given in [19].

${ }^{2}$ The offered traffic is the average call arrival rate (in calls per second) multiplied by the average call duration (in seconds) and measured in units of Erlangs.
} 
there exist nonnegative integer vectors $u \in Z^{L}$ and $f \in Z^{P}$ such that.

$$
m \mathcal{A}+(w \quad f) \mathcal{B} \leq \Lambda \mathcal{C} .
$$

where,

$$
\begin{aligned}
& \mathcal{A}=\left(\begin{array}{ccc}
I_{Y \times X} & 0_{N \times P} & \left(0_{N}^{T}\right.
\end{array}\right), \\
& \mathcal{B}=\left(\begin{array}{ccc}
0_{L \times Y} & -I^{T} & 1_{L}^{T} \\
-A & I_{P \times P} & 0_{P}^{T}
\end{array}\right) .
\end{aligned}
$$

and

$$
\mathfrak{C}=\left(\begin{array}{lll}
0_{N} & 0_{P} & 1
\end{array}\right)
$$

This set of feasible states for the network is of the form described in $[16$, Section 6$] .{ }^{3}$

Using [16. Theorem 3.1], the carried traffic for any RWA algorithm, $R$, for this network, satisfies,

$$
\frac{1}{\Lambda} T_{R}(\rho \cdot p) \leq T_{0}(r \cdot p)
$$

where $r=p / A$ and $T_{n}(r, p)$ is the value of the following linear program.

$$
T_{i}(r, p)=\max \sum_{i=1}^{N} s_{i}
$$

subject to

$$
\begin{array}{r}
s \cdot \mathcal{A}+t \mathcal{B} \leq \mathcal{C}, \\
s \leq p r .
\end{array}
$$

(This is just the $T_{i}^{\mathbf{A}}(\rho, p)$ program normalized by dividing the objective function and each of the constraints by $\Lambda$ and dropping the integrality constraints.)

The corresponding circuit-switching LP, obtained by normalizing the objective function and each of the constraints in the $U,(\rho, p)$ program by $\Lambda$ and dropping the integrality constraints, is the following.

$$
T_{i}(r, \mu)=\max \sum_{i=1}^{x} s_{i}
$$

subject to

$$
\begin{aligned}
s & \leq t A . \\
t B & \leq 1_{M} . \\
s & \leq p r .
\end{aligned}
$$

From [16. Theorem 2.1], $T_{0}(r, p)$ and $T_{c}(r, p)$ are continuous, nondecreasing, convex $\cap$ functions of $r$. Moreover, the $T_{w}(r, p)$ and $T_{r}(r, p)$ programs are parametric linear programs with parameter $r$ in the sense of [20, Section 1.9] and hence. $T,(r, p)$ and $T_{r}(r, p)$ are piecewise-linear functions of $r[20$, p. 701 .

The blocking probability, $B_{R}(\rho, p)$ for any RWA algorithm, $R$. is related to its carried traffic by

$$
T_{R}(\rho . p)=\rho\left(1-B_{R}(\rho, p)\right)
$$

\footnotetext{
"We have used matrix and vector notation here in lieu of stating the constraints as in the earlier II.P formulation to make it consistent with the notation used in $|16|$.
}

Therefore,

$$
B_{o}(r, p)=1-\frac{T_{o}(r \cdot p)}{r}
$$

and

$$
B_{c}(r \cdot p)=1-\frac{T_{c}(r \cdot p)}{r}
$$

are lower bounds for the blocking probability of any RWA algorithm in the wavelength-routing and circuit-switching cases. respectively.

We now show that $T_{o}$ and $T_{c}$ (or equivalently $B_{o}$ and $B_{c}$ ) are achievable when the number of wavelengths (circuits) is large.

\section{Asymptotic Optimality of $T_{0}(r, p)$ and $T_{c}(r, p)$}

Let $s=m^{\prime}, t=\left(w^{\prime} f^{\prime}\right)$ yield an optimal solution to the $T_{o}(r, p)$ LP. Consider a fixed RWA algorithm $F$, that assigns the fixed set of wavelengths $w_{i}=\left\lfloor w_{i}^{\prime} \Lambda\right\rfloor$ to each path in independent set $i$. Let $T_{\vec{F}}^{+}(\rho, p)$ denote the carried traffic for this algorithm.

1) The Deterministic Case: The asymptotic optimality of this fixed RWA algorithm in the deterministic case follows essentially from the fact that appropriately rounding any optimal solution to the $T_{0}(r . p)$ program yields a feasible solution to the $T_{i}^{1}(\rho . p)$ ILP whose value is asymptotically optimal (if $\Lambda$ is large and $r=\rho / A$ is fixed). We now prove this formally.

Lemma 3: Let $s=m^{\prime}$ and $t=\left(u^{\prime} f^{\prime}\right)$ constitute an optimal solution to the $T_{o}(r, p)$ linear program. Then $w_{i}=\left\lfloor w_{i}^{\prime} \Lambda\right\rfloor$. $f_{i}=\max \left(\left\lfloor f_{i}^{\prime} \Lambda\right\rfloor-L .0\right) . m_{i}=\max \left(\left\lfloor m_{i}^{\prime} \Lambda\right\rfloor-L P-P .(0)\right.$ yield a feasible solution to the $T_{0}^{1}(\rho . p)$ integer linear program.

Proof: Since s.t constitute a feasible solution to the $T_{0}(r . p)$ LP, we must have $\sum_{j=1}^{L} d_{i j} w_{j}^{\prime} \geq f_{i}^{\prime}$ and $\sum_{i=1}^{P} a_{i j} f_{j}^{\prime} \geq m_{i}^{\prime}$. We now show that similar inequalities are satisfied by $u_{i} . f_{i}$ and $m_{i}$.

We have obviously $\sum_{j=1}^{L} d_{i j} u_{j} \geq 0$. Furthermore,

$$
\begin{aligned}
\sum_{j=1}^{L} d_{i j} w_{j} & \geq \sum_{j} d_{i j}\left\lfloor w_{j}^{\prime} \Lambda\right\rfloor \\
& \geq \sum_{j} d_{i j}\left(w_{j}^{\prime} \Lambda-1\right) \\
& \geq\left\lfloor\sum_{j} d_{i j} w_{j}^{\prime} \Lambda\right\rfloor-L \\
& \geq\left\lfloor f_{i}^{\prime} \Lambda\right\rfloor-L
\end{aligned}
$$

where we have used the fact that $\sum_{j} d_{i j} \leq L$. Therefore

$$
\sum_{j} d_{i j} w_{j} \geq f_{i}
$$


Similarly $\sum_{i=1}^{P} f_{i} a_{i j} \geq 0$. Furthermore

$$
\begin{aligned}
\sum_{i=1}^{P} f_{i} a_{i j} & \geq \sum_{i}\left(\left\lfloor f_{i}^{\prime} \Lambda\right\rfloor-L\right) a_{i j} \\
& =\sum_{i} a_{i j}\left\lfloor f_{i}^{\prime} \Lambda\right\rfloor-L \sum_{i} a_{i j} \\
& \geq \sum_{i} a_{i j}\left(f_{i}^{\prime} \Lambda-1\right)-L P \\
& \geq \sum_{i}\left\lfloor m_{i}^{\prime} \Lambda\right\rfloor-P-L P
\end{aligned}
$$

where we have used the fact that $\sum_{i} a_{i j} \leq P$. Therefore,

$$
\sum_{i} f_{i} a_{i j} \geq m_{i}
$$

For the fixed routing algorithm $F$, using the above values of $m_{i}, f_{i}$, and $w_{i}$, we have

$$
\begin{aligned}
\frac{T_{F}^{\Lambda}(\rho, p)}{\Lambda} & =\frac{1}{\Lambda} \sum_{i} m_{i} \\
& \geq \frac{1}{\Lambda}\left(\sum_{i}\left\lfloor m_{i}^{\prime} \Lambda\right\rfloor-L P-P\right) \\
& \geq T_{o}(r, p)-\frac{1}{\Lambda}-\frac{L P}{\Lambda}-\frac{P}{\Lambda}
\end{aligned}
$$

Also

$$
\frac{T_{F}^{\Lambda}(\rho, p)}{\Lambda}=\frac{1}{\Lambda} \sum_{i} m_{i} \leq \sum_{i} m_{i}^{\prime} \leq T_{o}(r, p) .
$$

From the above we have the following proposition.

Proposition 2:

$$
\lim _{\Lambda \rightarrow \infty} \frac{1}{\Lambda} T_{F}^{\Lambda}(\rho, p)=T_{\mathrm{o}}(r, p) .
$$

2) The Random Case: The fixed RWA algorithm is a greedy algorithm in the following sense. It accepts a connection request between an $s-d$ pair if there is a free wavelength on any of the paths between that $s-d$ pair and rejects the connection request otherwise. Using [16, Theorem $4.1]$ if the offered traffics to different $s-d$ pairs are independent of one another, and if the traffic model satisfies the asymptotic traffic property (ATP) [16], this fixed RWA algorithm, $F$, satisfies,

$$
\lim _{\Lambda \rightarrow \infty} \frac{1}{\Lambda} T_{F}^{\Lambda}(\rho, p)=T_{o}(r, p),
$$

i.e., this fixed RWA algorithm is asymptotically optimal.

The ATP can be stated by considering a network with a single $s-d$ pair with an offered traffic of $\rho$ Erlangs and a fixed RWA algorithm that assigns $\Lambda$ channels to this $s-d$ pair and blocks a connection request if and only if all the $\Lambda$ channels are occupied. Let $T_{F}(\rho, 1, \Lambda)$ denote the carried traffic for this fixed RWA algorithm. Then, the statistics of the offered traffic are said to satisfy the ATP if,

$$
\lim _{\Lambda \rightarrow \infty} \frac{T_{F}(r \Lambda, 1, \Lambda)}{\Lambda}=\min (r, 1)
$$

Informally, the ATP is a kind of law of large numbers and simply states that, for a single $s-d$ pair, if the offered traffic exceeds the number of available wavelengths, all the available wavelengths are asymptotically occupied, and if the offered traffic is less than the number of wavelengths, all connection requests are asymptotically honored. The ATP is satisfied by many common traffic models including Poisson arrivals [16]. It was shown in [16] that a similar fixed routing algorithm is asymptotically optimal for the circuit-switched case.

\section{$E$. Some Simple Bounds on $T_{o}(r, p)$ and $T_{c}(r, p)$}

The following observations are useful in deriving the values of $T_{o}(r, p)$ and $T_{c}(r, p)$ for simple networks.

Proposition 3: Let $K$ be the maximum number of edge disjoint paths in a graph $G$, i.e., $K$ is the cardinality of the maximum independent set in the path graph $G_{p}$. Then $T_{o}(r, p) \leq K$.

Proof: Since paths in $G$ that use the same wavelength must be edge-disjoint, the maximum number of times a wavelength can be used is the maximum number of edgedisjoint paths in $G$; hence, $T_{o}(r, p) \leq K$.

Proposition 4: Let $E$ be the total number of links in a circuit-switched network $G$. Let $H$ denote the minimum number of edges in a shortest-path between all desired sourcedestination pairs between which there is nonzero traffic. Then $T_{c}(r, p) \leq E / H$.

Proof: Since a connection takes up at least $H$ circuits, and there are a total of $E \Lambda$ circuits, the total number of connections that can be supported is $\leq E \Lambda / H$. Thus the maximum number of connections that can be supported per wavelength, $T_{\mathrm{c}}(r, p) \leq E / H$.

Definition: We define the reuse factor at a blocking probability $b$ of a network $G$ for a given path-s-d-pair incidence matrix $A$ and traffic pattern vector $p$, under the operation of a routing and wavelength assignment algorithm, $X, R_{A, p}^{X, b}(G)$, to be the maximum offered traffic per wavelength so that the blocking probability is $\leq b$. In the simulation results that we will present below, we will consider the reuse factor at a blocking probability of $1 \%$.

We define the asymptotic reuse factor $R_{A, p}(G)$ of a network $G$ for given $A$ and $p$ as the maximum offered traffic per wavelength for which the blocking probability can be made arbitrarily small if the number of wavelengths is sufficiently large.

Proposition 5: Consider a circuit-switched network $G$ with exactly one path between every s-d pair (i.e., $A$ is the identity matrix, $I)$. Let $\rho$ denote the total offered traffic to such a network $G$ with traffic pattern vector $p$. Let $\rho_{j}$ denote the offered traffic to link $j$ in the network and let $\rho_{\max }=\max _{j} \rho_{j}$. Then,

$$
R_{I, p}(G)=\frac{\rho}{\rho_{\max }} .
$$

Proof: It can be checked that $s_{i}=t_{i}=p_{i} r$ is a feasible solution to the $T_{c}(r, p)$ LP for $r \leq \rho / \rho_{\max }$ and is also optimal because of the constraints $s \leq p r$, i.e., $T_{c}(r, p)=r$ for $r \leq \rho / \rho_{\max }$. Therefore, $R_{I, p}(G) \geq \rho / \rho_{\max }$. Furthermore, the constraints $s \leq t I=t$ and $t B \leq 1_{M}$ imply that $s_{i}=p_{i} r$ is not a feasible solution for $r>\rho / \rho_{\max }$, and thus $T_{c}(r, p)<r$ for $r>\rho / \rho_{\max }$. Therefore, $R_{I, p}(G)=\rho / \rho_{\max }$. 


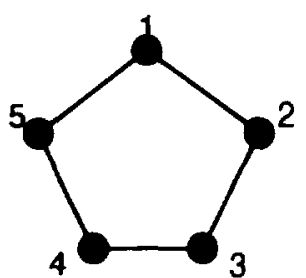

Fig. 4. A five node network.

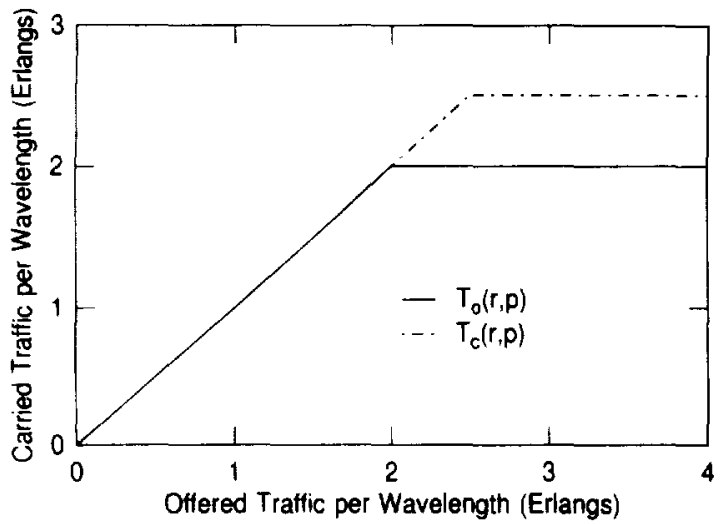

Fig. 5. Carried traffic versus offered traffic for the above five-node network. $T_{1}(r, p)$ is the upper bound without wavelength converters and $T_{r}(r . p)$ is the upper bound with wavelength converters

\section{F. A Heuristic RWA Algorithm}

Consider the following shortest-path RWA (SP-RWA) algorithm. The set of shortest paths between a source-destination pair is ordered in some manner. The set of wavelengths is ordered in some manner. A new connection is routed on the first path on which a wavelength is available. Among the set of available wavelengths on that path, the first one is selected. If no path can be found, the connection is considered blocked.

We simulated the performance of this simple algorithm for traffic where connection requests are assumed to arrive according to a Poisson process and last for a duration that is exponentially distributed

\section{EXAMPLES}

To illustrate the use of our bound, we provide two examples. The first is a simple 5-node network that brings out the difference between the circuit-switched network and the optical network. The second is a more complicated 20 -node network and is intended to demonstrate the application of our results to more realistic networks.

\section{A. A Simple Example}

Consider the 5-node pentagon network $C_{5}$ shown in Fig. 4. Let the source-destination pairs of interest, indexed from 1 to 5 , be $(1,3),(2,4),(3,5),(4,1)$, and $(5,2)$, i.e., each node communicates with two other nodes in the network. The paths of interest are then 123, 1543, 234, 2154, 345, 3215, $451,4321,512,5432$ and will be indexed from 1 to 10. The maximal independent sets in the path graph are $\{1,2\}$, $\{3,4\},\{5,6\},\{7,8\},\{9,10\},\{1,7\},\{3,9\},\{5,1\},\{7$, 3\}. $\{9,5\}$. For simplicity, consider the uniform traffic case,

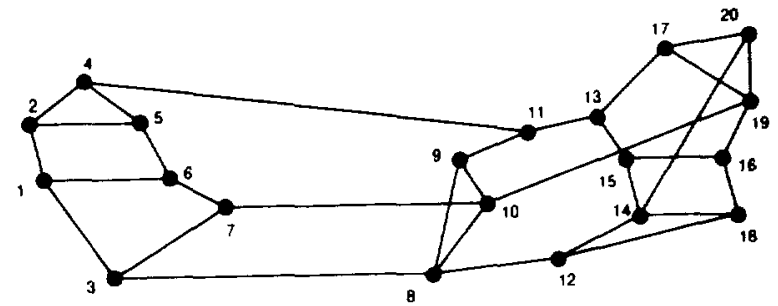

Fig. 6. A 20-node network representing a skeleton of the original Arpanet.

i.e., $p_{i}=1 / 5, i=1, \cdots, 5$. In this case, observe that the maximum cardinality of an independent set is 2 and hence from Proposition 3, $T_{o}(r, p) \leq 2$. The shortest-path has two links $(H=2)$ and there are $E=5$ links in the graph; hence from Proposition 4, we get $T_{c}(r, p) \leq 5 / 2$. Solving the linear programs yield,

$$
T_{o}(r . p)= \begin{cases}r, & 0 \leq r \leq 2 \\ 2, & r>2\end{cases}
$$

and

$$
T_{c}(r, p)= \begin{cases}r, & 0 \leq r \leq 5 / 2 \\ 5 / 2, & r>5 / 2\end{cases}
$$

and hence the upper bounds of Propositions 3 and 4 are tight for large $r$ in this example. Fig. 5 plots $T_{o}(r, p)$ and $T_{c}(r, p)$ as a function of $r$.

An asymptotically optimal fixed RWA algorithm for the optical network assigns the first $\Lambda / 5$ wavelengths to both paths between $s-d$ pair 1 , the second $\Lambda / 5$ wavelengths to both paths between $s$ - $d$ pair 2, etc. For $r \geq 2$, this algorithm realizes a total carried traffic of $2 \Lambda$ asymptotically, thus achieving the bound of Proposition 3.

An asymptotically optimal fixed RWA algorithm for the circuit-switched network assigns $\Lambda / 2$ circuits for connections between each $s-d$ pair. All connections are routed on the unique shortest paths. For $r \geq 5 / 2$, this algorithm realizes a total carried traffic of $5 \Lambda / 2$ asymptotically, thus achieving the bound of Proposition 4.

\section{B. A Larger Example}

Consider the 20-node skeleton of the Arpanet shown in Fig. 6 from [21, p. 138]. For this network we consider the class of algorithms that use only shortest paths. Let the $s-d$ pairs of interest be $\{1,13\},\{2,7\},\{3,15\},\{6,8\},\{11,14\}$, $\{4,20\},\{5,19\},\{9,18\},\{10,17\},\{12,16\}$. In this example there are 14 shortest paths: 3 shortest paths between $s$ - $d$ pairs $\{2,7\}$ and $\{6,8\}$ and a single shortest path between the other 8 $s-d$ pairs. The path graph consists of 14 nodes corresponding to these shortest paths and has $\mathbf{4 3}$ maximal independent sets.

Consider first the uniform traffic case. Fig. 7 shows the carried traffic and blocking probability as a function of offered traffic. In this case,

$$
T_{c}(r \cdot p)=T_{o}(r, p)=\min (r .7 r / 10+1,3 r / 10+3,6) .
$$

The performance of the fixed RWA algorithm of Section II-D and the simulated performance of the heuristic algorithm of Section II-F are also indicated. 


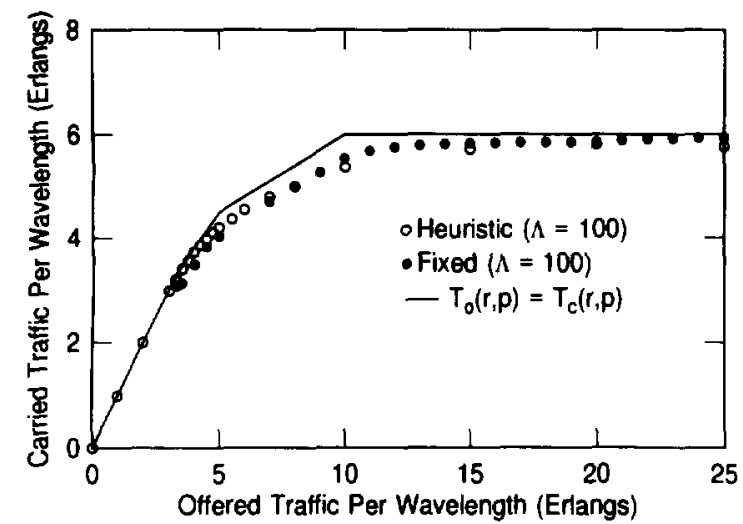

(a)

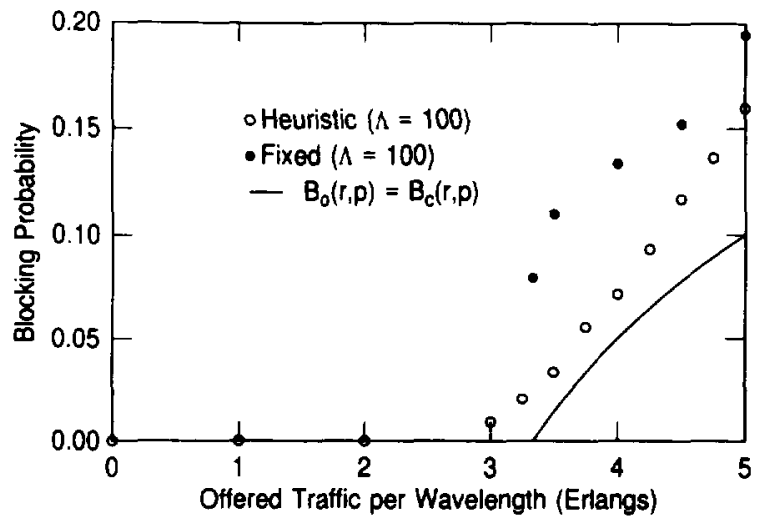

(c)

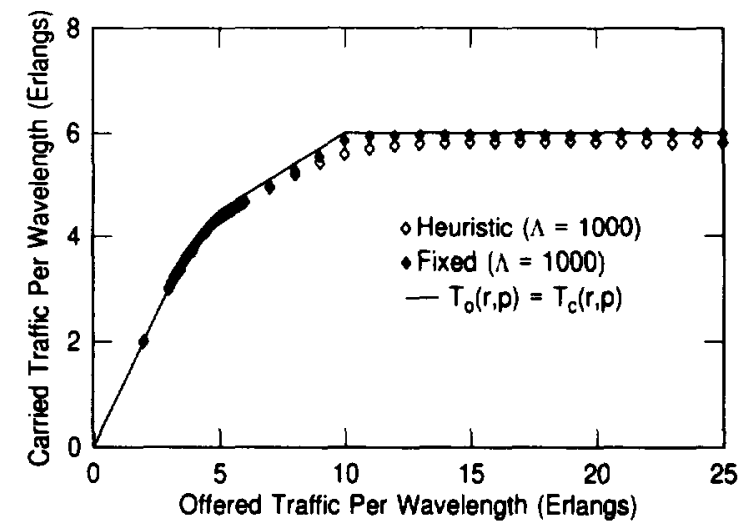

(b)

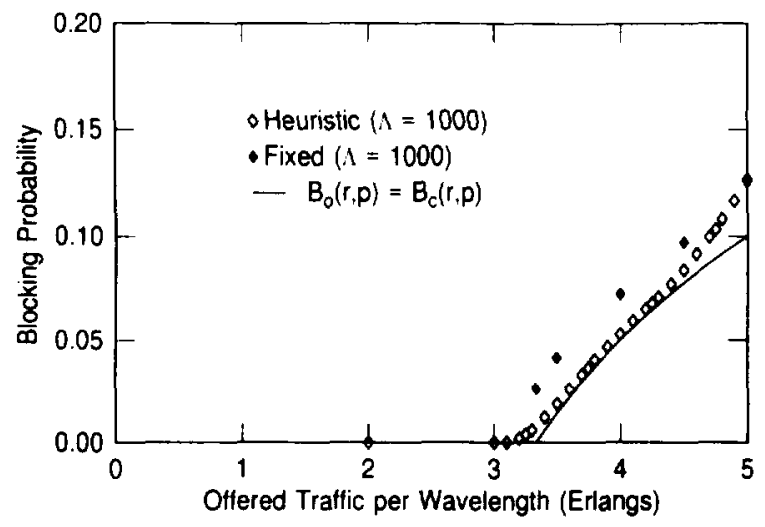

(d)

Fig. 7. Carried traffic and blocking probability versus offered traffic for the 20-node network, assuming uniform traffic. $T_{0}(r . p)$ is the upper bound on carried traffic without wavelength converters and $T_{c}(r, p)$ is the upper bound with wavelength converters. $B_{o}(r . p)$ is the corresponding lower bound on blocking probability without wavelength converters and $B_{c}(r, p)$ is the lower bound with wavelength converters.

Remark: For this example, instead of just shortest paths, if we consider all paths with five or fewer hops, there are a total of 63 paths between the desired source-destination pairs, and there are 6865 maximal independent sets in the corresponding path graph, illustrating the exponential growth in the number of independent sets as a function of the number of nodes in the path graph. The carried traffic in the network can be improved if we route over these paths. Specifically, in this case,

$$
T_{c}(r, p)=T_{o}(r, p)=\min (r, 3 r / 10+3,2 r / 10+4,7) .
$$

Surprisingly, if we consider all paths with eleven or fewer hops (there are 2038 such paths), $T_{c}(r, p)$ does not increase further, implying $T_{o}(r, p)$ does not increase either (since $\left.T_{o}(r, p) \leq T_{c}(r, p)\right)$. This suggests that, at least for a large number of wavelengths, more complicated routing algorithms that consider paths with many hops may not help improve the carried traffic. We are investigating this further.

Now consider the case of nonuniform traffic with the (arbitrarily chosen) vector

$$
p=(2 / 23,6 / 23,2 / 23,6 / 23,2 / 23,1 / 23,
$$

Fig. 8 shows the carried traffic and blocking probability as a function of offered traffic. In this case,

$$
T_{c}(r, p)=\min (r, 10 r / 23+2,5 r / 23+3,2 r / 23+4,6)
$$

and

$$
T_{o}(r, p)=\min (r, 10 r / 23+2,4 r / 23+3,2 r / 23+4,6) .
$$

Figs. 7 and 8 also illustrate the asymptotic optimality of the fixed RWA algorithm for large $\Lambda$ proved in Section II-D. However, this is a different (but fixed) RWA algorithm for each value of the offered traffic. Therefore, the implementation of the fixed RWA algorithm requires that the offered traffic be known and since in practice the offered traffic may be unknown and changing, this is an impractical algorithm.

In this case, an RWA algorithm whose implementation does not require an estimate of the offered traffic is desirable. The SP-RWA algorithm of Section II-F has this property and it is gratifying to note, from Figs. 7 and 8, that its performance is close to that achievable by any ${ }^{4}$ RWA algorithm in this example (for both the uniform and nonuniform cases). Moreover, we would not have been able to draw this conclusion in the nonuniform case, if our tighter upper bound had not been available.

\footnotetext{
${ }^{4}$ Note that even though an RWA algorithm has no choice regarding the path on which to route the connection for most of the $s-d$ pairs in this example, it still has the choice of deliberately blocking the connection request and the task of choosing one of the (potentially) many available wavelengths on this path. Even if there were a fixed path between every $s-d$ pair in the network, a number of RWA heuristics can be designed simply based on the way in which they pick one of the available wavelengths on this path-least used, most used. etc. [7].
} 


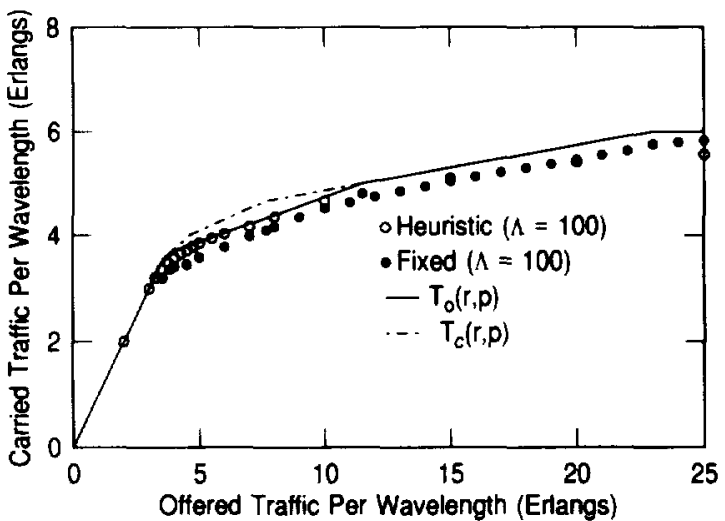

(a)

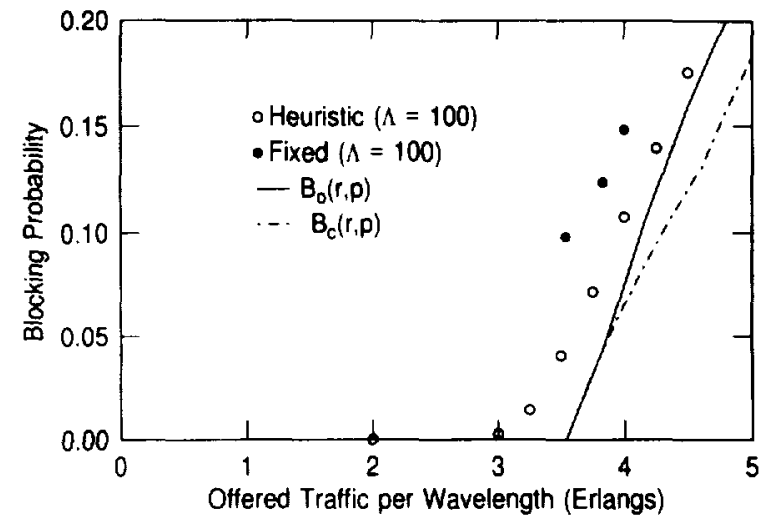

(c)

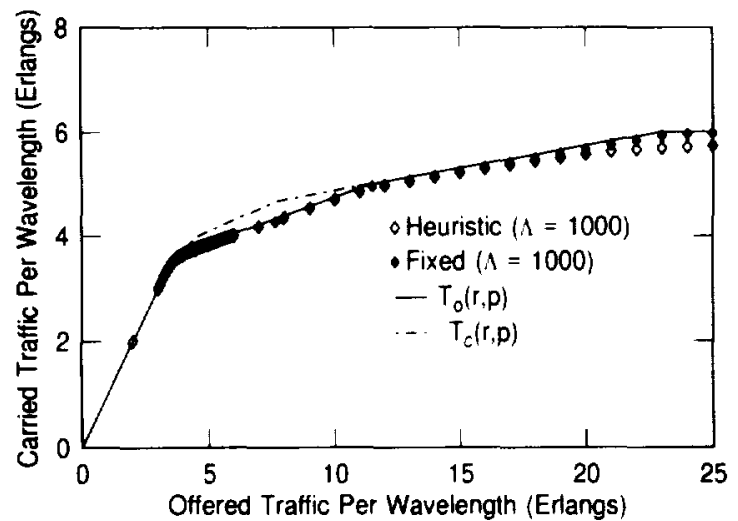

(b)

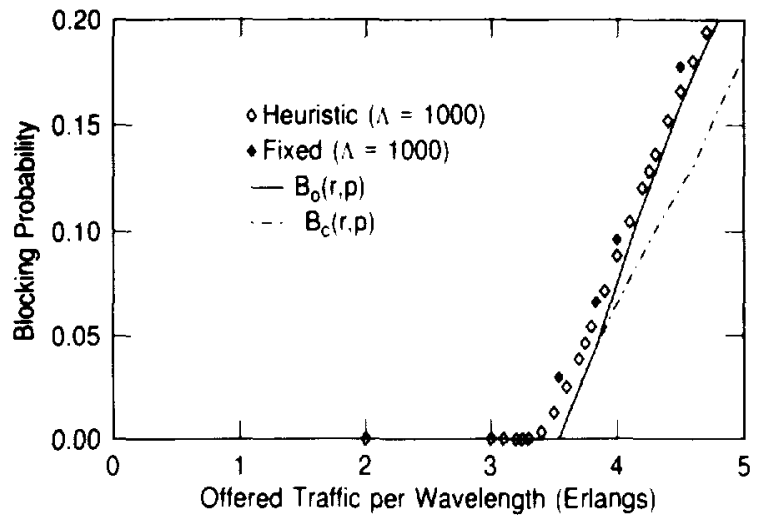

(d)

Fig. 8. Carried traffic and blocking probability versus offered traffic for the 20-node network, for the nonuniform traffic case.

It must also be noted that since a fixed RWA algorithm implies a fixed setting of the switches shown in Fig. 2, if the mean offered traffic is known and not changing, the network can be implemented without optical switches. Thus the principal advantage of optical switching in an all-optical network is probably the ability to adapt to the unknown traffic and not just the amount of wavelength reuse that can be obtained.

The blocking probability curves shown in Figs. 7 and 8 can be plotted in different ways to illustrate the effect of different parameters. For instance Fig. 9 shows the blocking probability plotted against the number of wavelengths for the nonuniform traffic case, for a total offered load of 40 erlangs (i.e., an average of 4 full-duplex connections per node). It is also possible to recast the results in Figs. 7 and 8 to plot the blocking probability as a function of the offered (or carried) traffic for a fixed number of wavelengths.

\section{WaVELENGTH ReUSE IN LARGE NeTwORKS}

We now turn to the question of determining the wavelength reuse factor $R$ for large networks with and without wavelength converters. One way to compare the performance of different topologies is to compare the values of $R$ for uniform traffic. Using Proposition 5, we can easily compute the asymptotic value of $R$ for any given RWA algorithm and use that as a measure to compare different topologies. Although Proposition 5 gives a bound on $R$ for a circuit-switched network (with

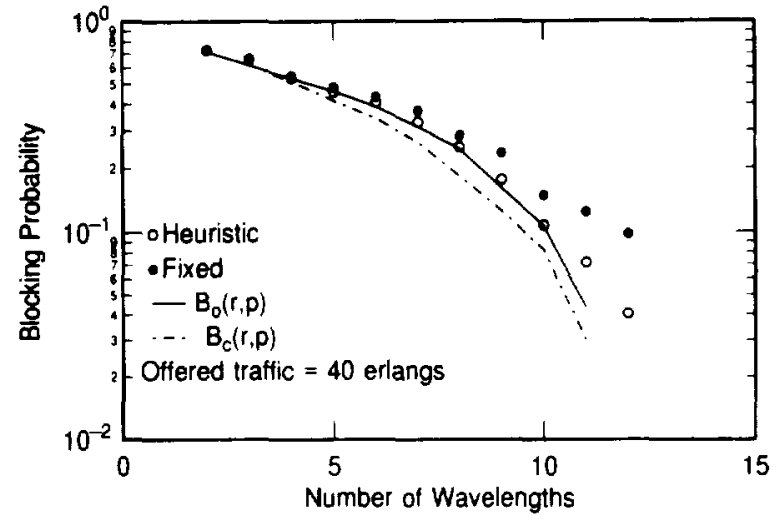

Fig. 9. Blocking probability versus number of wavelengths for the 20 -node network, for the nonuniform traffic case. The total offered traffic is 40 erlangs.

wavelength converters), our simulation results below show that the reuse factors with and without wavelength converters are strongly correlated.

In order to determine $R$ when the number of wavelengths is limited (in practice), we studied the performance of the SP-RWA algorithm of Section II-F for many topologies via simulation assuming a uniform traffic distribution over all possible source-destination pairs, with Poisson arrivals and exponential holding times for connections. A $1 \%$ blocking probability requirement was assumed. 


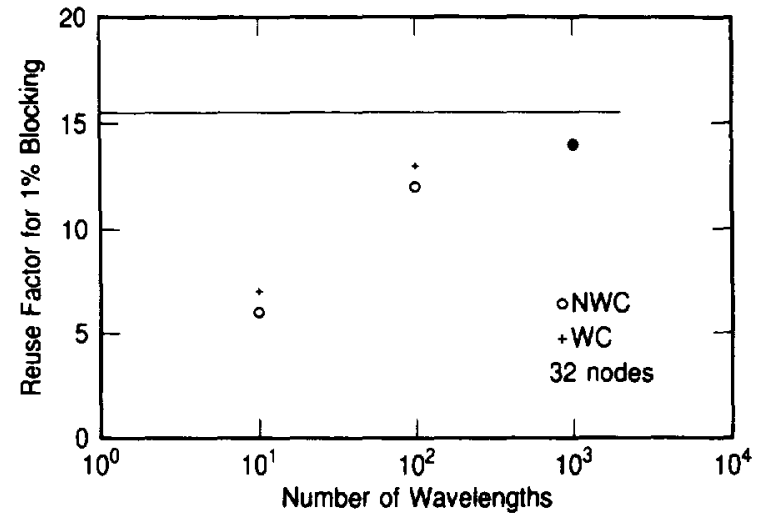

Fig. 10. Reuse factor plotted against the number of wavelengths for a 32-node random graph with average degree 4 , with wavelength converters (WC) and without converters (NWC). The horizontal line indicates the value of our bound for circuit-switching, $T_{c}$, for $1 \%$ blocking.

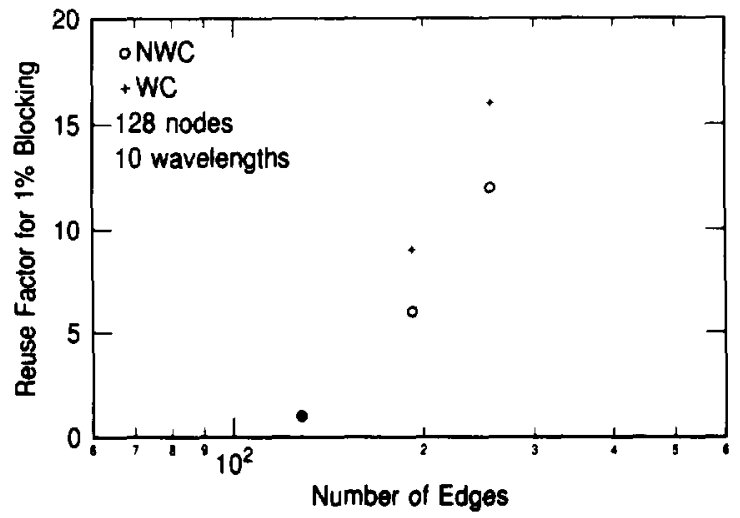

Fig. 11. Reuse factor plotted against the number of edges for a number of 128-node random graphs, with wavelength converters (WC) and without converters (NWC).

Fig. 10 shows the reuse factor $R$ plotted against the number of wavelengths for a 32-node random graph with average degree 4. (To generate a random graph with $n$ nodes and average degree $\Delta$, we first place $n$ edges to create a cycle and then, for each of the remaining $n(\Delta / 2-1)$ edges we choose, in succession, a pair of nodes randomly from the node pairs that are not connected by an edge.) Also shown is our circuit-switched network bound $\left(T_{c}\right)$ with one shortest path for every $s-d$ pair. We expect the reuse factor to increase with the number of wavelengths due to the effect of trunking efficiency, i.e., the phenomenon whereby if both the traffic and the link capacities are scaled proportionally in a circuitswitched system, the blocking probability is reduced. For the case of Poisson arrivals on a link, this property can easily be proved using the Erlang-B formula [22, p. 147]. The figure verifies that this is indeed the case for networks and also that the reuse factor is close to the asymptotic reuse factor when 1000 wavelengths are available. We observed a similar behavior with our other sample networks as well.

Fig. 11 shows the reuse factor $R$ plotted against the number of edges for a number of 128-node random graphs. Clearly, increasing the number of edges reduces the average path length between nodes and hence improves the reuse factor.

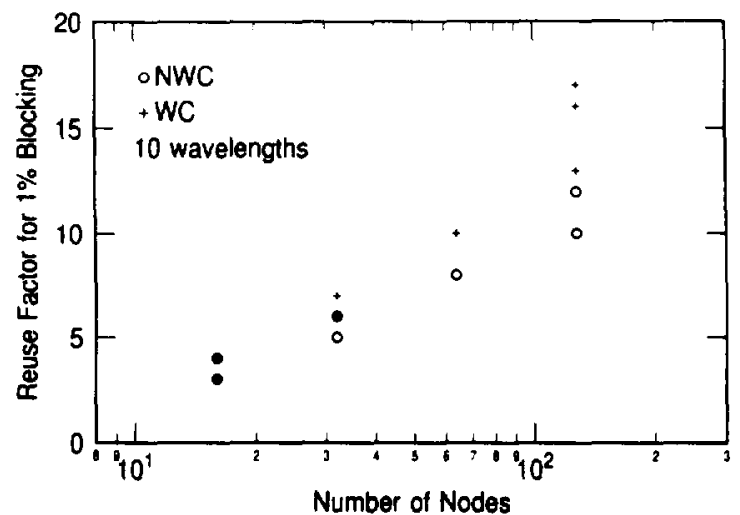

Fig. 12. Reuse factor plotted against the number of nodes for random graphs with average degree 4 , with wavelength converters (WC) and without converters (NWC).

Fig. 12 shows the reuse factor $R$ plotted against the number of nodes, $n$. For each value of $n$, we obtained $R$ via simulation for three different random graphs, each of average degree 4 . In addition we have also simulated two 1000-node random networks of average degree 4 , but with uniform traffic over only 5000 randomly chosen source-destination pairs. The reuse factors for these two networks were 43 and 49 , respectively, without wavelength converters, and 58 and 68 , respectively, with wavelength converters, assuming 10 wavelengths are available. These results show that 1$) R$ increases with $n$, and 2) the difference in performance between having and not having wavelength converters increases with $n$ (almost no difference for 16 node networks and up to a $40 \%$ difference for the 1000 node networks considered here). The reason for the former is that the average path length in the network grows roughly as $\log n$, while the number of edges grows as $n$ and thus we would expect the reuse factor to grow roughly as $n / \log n$. However, we do not yet have an intuitive explanation as to why 2) should be true.

Finally, a wide class of regular networks such as shufflenets [23] and deBruijn graphs [24] have been studied as possible topologies for optical networks. An (undirected) deBruijn graph of degree $\Delta$ and diameter $D$ has $(\Delta / 2)^{D}$ nodes. corresponding to the state transition diagram of a $D$-digit shift register with each digit $\in\{0,1, \cdots, \Delta-1\}$. Fig. 13 shows the reuse factor $R$ plotted against the number of nodes, $n$ for deBruijn graphs and random graphs. For each value of $n$, we use a deBruijn graph of degree 4 and and a random graph with average degree 4 . The figure shows that for moderate number of nodes (16-64) there is no advantage to using deBruijn graphs, but for larger networks (128 and above), deBruijn graphs appear to perform better, implying that careful design of topology is not as important when the number of nodes is small for uniform traffic. When the traffic is known to be nonuniform, then signficant benefits can be obtained by proper topology design.

\section{AN Alternate Routing Node ARChItecture}

So far, we have assumed that in networks without wavelength converters, two connections can be assigned the same wavelength provided they do not share any link in the network. 


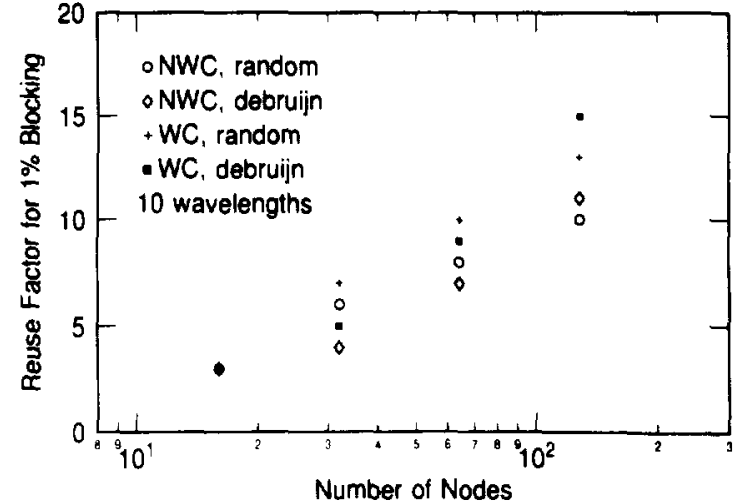

Fig. 13. Reuse factor plotted against the number of nodes for deBruijn graphs with degree 4 and random graphs with average degree 4 , with wavelength converters (WC) and without converters (NWC).

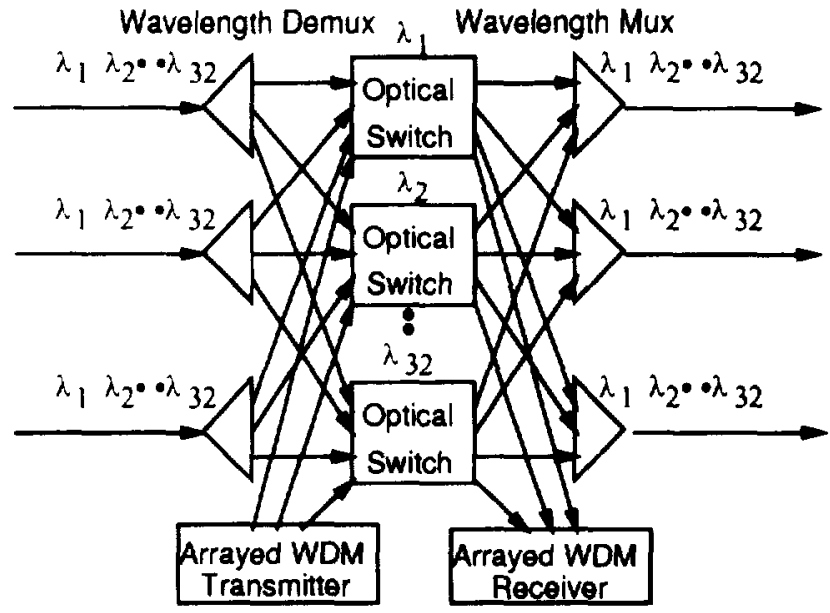

Local Source and Sink

Fig. 14. Structure of a simplified reconfigurable routing node.

Implicitly, this also assumes that two connections emanating from a node can be at the same wavelength provided they are routed on different links out of the node. The optical hardware required to realize this is shown in Fig. 2. An arrayed multiwavelength transmitter and an arrayed multiwavelength receiver are required for each link at the node. The amount of hardware required can be reduced by using the node structure shown in Fig. 14. However, this structure imposes an additional constraint on the RWA problem in that two connections emanating from a given node must be assigned different wavelengths. Clearly this reduces the reuse factor. Fig. 15 shows the reduction in reuse factor due to this added constraint.

\section{CONCLUSION}

Although the bound presented in this paper can be obtained by solving a linear program, the number of variables in the linear program may be an exponential function of the number of source-destination pairs in the case of the optical network without wavelength converters. However, since the bound is on a per-wavelength basis, it need be computed only once and can then be scaled easily with the number of wavelengths. Moreover, the bound is achieved by a fixed-routing algorithm

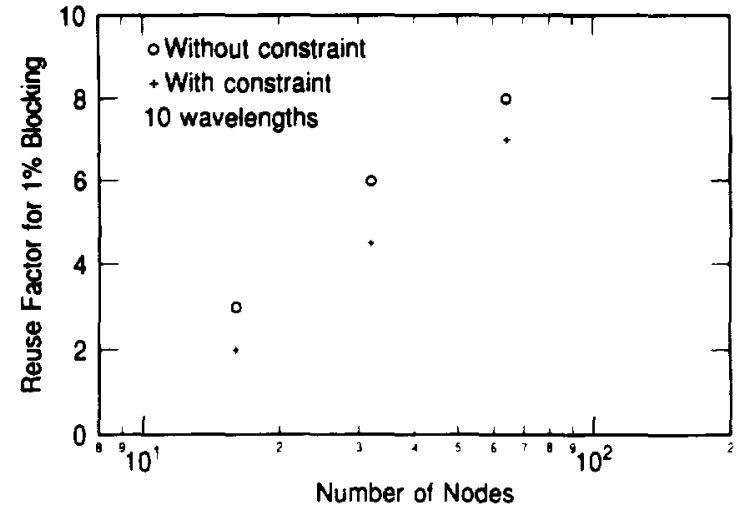

Fig. 15. Reuse factor plotted against the number of nodes for random networks with average degree 4 , with and without the constraint that two connections out of a given node must be assigned different wavelengths.

TABLE I

Illustrative Reuse Factors for Sample Random Networks, Each of Average Degree 4 for Uniform Traffic and the Simple RWA Heuristic Without Wavelength Converters

\begin{tabular}{cccc}
\hline $\begin{array}{c}\text { No. of } \\
\text { nodes }\end{array}$ & $\begin{array}{c}\text { Wave- } \\
\text { lengths }\end{array}$ & $\begin{array}{c}\text { Reuse } \\
\text { factor }\end{array}$ & $\begin{array}{c}\text { Connections } \\
\text { per node }\end{array}$ \\
\hline 128 & 10 & 12 & 2 \\
128 & 32 & 20 & 10 \\
1000 & 10 & 58 & 1 \\
1000 & 32 & 78 & 5 \\
\hline
\end{tabular}

asymptotically (when the number of wavelengths is large). It can be used as a metric against which the performance of different heuristics can be compared. Moreover, solving the LP enables us to determine the assignment of paths and wavelengths used by an asymptotically optimal fixed RWA algorithm. This information can be useful in indicating which links are congested and how the network can be modified by the addition of more links/wavelengths to relieve this congestion and improve its performance.

Using two examples, we showed that this bound yields a better bound on the carried traffic than the bound for the corresponding circuit-switched network, or equivalently the corresponding optical network using dynamic wavelength converters. Indeed using this bound, we showed in the second example that the performance of the simple SP-RWA heuristic described here, assuming Poisson arrivals and exponential holding times, is very close to that of an optimal algorithm.

We then quantified the reuse factor for larger networks as a function of the number of nodes, edges, and wavelengths via simulation. Table I provides some illustrative results for sample networks. Based on the results, we can infer the following:

1) We can build large all-optical networks without wavelength converters and support a modest number of connections per node with a reasonable number of wavelengths. For instance in the 128-node network considered in Table I we can obtain a reuse factor of 20 when 32 wavelengths are available, assuming uniform traffic and using the simple RWA heuristic. This indicates that with 32 wavelengths, we can suppon an average of 10 all-optical full-duplex connections per 
node. Another example from Table I shows that we can support over 2500 connections, or approximately 5 connections per node in a 1000 -node random network with average degree 4 using 32 wavelengths.

2) The results also show that wavelength converters offer a $10-40 \%$ increase in the amount of reuse achievable for our sampling of 14 networks ranging from 16 to 1000 nodes when the number of wavelengths available is small (10 or 32). They appear to help more for large networks than for small ones, or when the number of wavelengths available is small.

Although the difference in blocking performance between networks with and without wavelength converters was not very large for all the network examples considered here, it is possible to construct networks where the difference is large. This can be done by ensuring that there is a large set of paths that form a clique in the path graph (i.e., any two paths in this set share some link in the network), but choosing them so that few very paths share any single link. An example given in [12] shows such a network and traffic pattern that can be supported without blocking by a small constant number of wavelengths if wavelength converters are available, but requires as many wavelengths as the number of nodes when wavelength converters are not available.

\section{REFERENCES}

[1] P. E. Green, Fiber-Optic Networks. Englewood Cliffs, NJ: Prentice-Hall, 1992.

[2] N. K. Cheung. G. Nosu and G. Winzer, eds., IEEE J. Select. Areas Commun., Special Issue on Dense WDM Networks, vol. 8, Aug. 1990.

[3] M. J. Karol, C. Lin, G. Hill, and K. Nosu, Eds., IEEE/OSA J. Lightwave Technol, Special Issue on Broadband Optical Networks, May/June 1993.

[4] R. Ramaswami, "Multi-wavelength lightwave networks for computer communication," IEEE Commun. Mag., vol. 31, pp. 78-88, Feb. 1993.

[5] I. Chlamtac, A. Ganz, and G. Karmi, "Purely optical networks for terabit communication," in Proc. IEEE INFOCOM '89, 1989, pp. 887-896.

[6] T. E. Stern, "Linear lightwave networks: How far can they go?", in Proc. GLOBECOM'90, 1990 , pp. 1866-1872.

[7] K. Bala, T. E. Stern, and K. Bala, "Algorithms for routing in a linear lightwave network," in Proc. INFOCOM'91, 1991, pp. 1-9.

[8] K. Bala, "Routing in linear lightwave Networks," Ph.D. dissertation, Columbia Univ., 1993, CTR Tech. Rep. 323-93-02.

[9] I. Chlamtac, A. Ganz, and G. Karmi, "Lightpath communications: An approach to high-bandwidth optical WAN's," IEEE Trans. Commun. vol. 40, pp. $1171-1182$, July 1992.

[10] R. A. Barry and P. A. Humblet, "On the number of wavelengths and switches in all-optical networks," IEEE Trans. Commun., vol. 42, pp. 583-591, Feb.Mar./Apr. 1994.

[11] R. K. Pankaj, "Architectures for linear lightwave networks," Ph.D. dissertation, Massachusetts Inst. of Technol., Cambridge, MA, 1992.

[12] A. Aggarwal, A. Bar-Noy, D. Coppersmith, R. Ramaswami, B. Schieber, and M. Sudan, "Efficient routing and scheduling algorithms for optical networks," in Proc. 5th Annu. ACM-SIAM Symp. Discrete Algorithms, Jan. 1994, pp. 412-423.
[13] I. Chlamtac, A. Ganz and G. Karmi, "Lightnets: Topologies for highspeed optical networks," IEEEJOSA J. Lightwave Technol., vol. 11, pp. 951-961, May/June 1993.

[14] B. Mukherjee, S. Ramamurthy, D. Banerjee and A. Mukherjee, "Some principles for designing a wide-area optical network," in Proc. IEEE INFOCOM'94, 1994.

[15] K.-C. Lee and V. O. K. Li, "A wavelength-convertible optical network," IEEEIOSA J. Lightwave Tech., vol. 11, pp. 962-970. May/June 1993.

[16] R. J. McEliece and K. N. Sivarajan, "Maximizing marginal revenue in generalized blocking service networks," in Proc. 30th Annu. Allerton Conf. Commun., Contr. Comput., vol. 18, pp. 455-464, 1992.

[17] F. P. Kelly, "Blocking probabilities in large circuit-switched networks," Adv. Appl. Prob., vol. 18, pp. 473-505, 1986.

[18] M. Gondran and M. Minoux, Graphs and Algorithms. New York: Wiley, 1986.

[19] C. Bron and J. Kerbosch, "Algorithm 457: Finding all cliques of an undirected graph," Commun. $A C M$, vol. 16, no. 9, pp. 575-577, 1973.

[20] J. N. Franklin, Methods of Mathematical Economics. New York: Springer-Verlag, 1980

[21] C. J. Colbourn, The Combinatorics of Network Reliability. New York: Oxford University Press, 1987.

[22] R. Syski, Introduction to Congestion Theory in Telephone Systems. Amsterdam, The Netherlands: North-Holland. 1986.

[23] M. G. Hluchyj and M. J. Karol, "Shufflenet: An application of generalized perfect shuffies to multihop lightwave networks," IEEEJOSA $J$ Lightwave Technol, vol. 9, no. 10, pp. 1386-1397, 1991.

[24] K. N. Sivarajan and R. Ramaswami, "Lightwave networks based on deBruijn graphs," IEEE/ACM Trans. Networking, vol. 2, pp. 70-79, Feb. 1994.

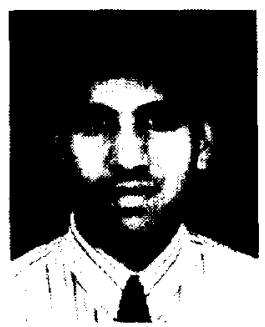

Rajiv Ramaswami (S'88-M'90) received the B.Tech. degree in electrical engineering (electronics) from the Indian Institute of Technology, Madras in 1986, and the M.S. and Ph.D. degrees in electrical engineering and computer science from the University of California, Berkeley in 1988 and 1990 , respectively.

In 1989 he joined the IBM T. J. Watson Research center as a staff member. He currently manages the optical network systems group.

Dr. Ramaswami is an Editor of the IEEE/ACM Transactions on Networking and a Guest Editor of a joint special issue on Optical Networks of the IEEE JOURNAL ON SELECTED AREAS IN Communication and the IEEE/OSA Journal of Lightwave TeChNOLOGY.

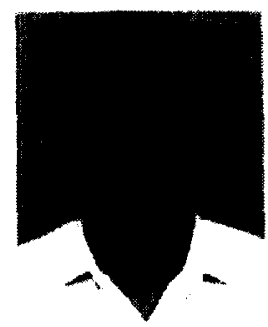

Kumar N. Sivarajan (S'88-M'91) received the B.Tech. degree in electrical engineering (electronics) from the Indian Institute of Technology, Madras in 1987, and the M.S. and Ph.D. degrees in electrical engineering from the California Institute of Technology in 1987 and 1990 , respectively.

Since July 1990 he has been with the IBM T. J. Watson Research Center, Yorktown Heights, NY Since October 1994, he has been with the Indian Institute of Science, Bangalore, on leave from IBM. His research interests are in the areas of optical, cellular, and ATM networks where he has worked on network architecture, algorithms, and performance analysis. 